PARÉ, Moussa : Aux origines des oppositions idéologiques dans la zone sahélienne. Les Almoravides dans le Bilad al-Sudan occidental au XI ${ }^{\mathrm{e}}$ siècle, in: Regards croisés Afrique-Europe 1 (2021), p. 59-71

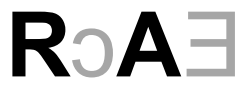

University of Bamberg Press - ISSN : 2749-8514

DOI: https://doi.org/10.20377/rcae-15

(finalisé: 30/11/2021 - publié: 15/12/2021)

MOUSSA PARÉ

\title{
Aux origines des oppositions idéologiques dans la zone sahélienne
}

\author{
Les Almoravides dans le Bilad al-Sudan occidental \\ au $\mathrm{XI}^{\mathrm{e}}$ siècle
}

\section{Résumé}

L'article « Aux origines des oppositions idéologiques dans la zone sahélienne. Les Almoravides dans le Bilad al-Sudan occidental au $\mathrm{XI}^{\mathrm{e}}$ siècle » analyse les raisons politiques et idéologiques de l'expansion almoravide dans le Bilad al-Sudan. Il ressort ainsi de l'analyse des sources narratives arabo-musulmanes que la volonté des émirs almoravides d'éradiquer les hérésies kharidjites d'une part, et d'autre part la fondation d'un vaste empire malékite dans le Dar al-Islam occidental ont motivé la poussée almoravide vers le Soudan Occidental. Ces desseins d'ordre idéologique et politique ont favorisé la diffusion d'un islam orthodoxe. Jusque-là le Bilad al-Sudan était traversé par des clivages ethniques, sociaux et religieux. L'islam s'y trouvait mêlé de tendances hétérodoxes : chiisme et surtout le kharidjisme. Le mouvement almoravide a réalisé cette union sous la bannière du malékisme triomphant.

Mots-clés: Almoravides, Aoudaghost, Bilad al-Sudan, kharidjisme, malékisme

\section{Zusammenfassung}

Der Aufsatz “Zu den Ursprüngen der ideologischen Gegensätze in der Sahelzone. Die Almoraviden im westlichen Bilad al-Sudan im 11. Jahrhundert" untersucht die politischen und ideologischen Gründe für die almoravidische Expansion in den Bilad al-Sudan. Die Analyse der arabisch-islamischen erzählenden Quellen zeigt, dass der almoravidische Vorstoß in den westlichen Sudan einerseits durch die Absicht der almoravidischen Emire,

catégorie: article original 
die kharidschitischen Häresien zu eliminieren, andererseits durch das Ziel der Gründung eines großen malikitischen Reiches im westlichen Dar al-Islam motiviert war. Diese ideologischen und politischen Ziele förderten die Verbreitung eines orthodoxen Islams. Bis dahin war der Bilad al-Sudan von ethnischer, sozialer und religiöser Vielfalt gekennzeichnet. Der Islam war dort durchsetzt mit heterodoxen Tendenzen: Schiismus und vor allem Kharidschismus. Die Bewegung der Almoraviden vollzog diese Vereinigung unter dem Banner des triumphierenden Malekismus.

Schlagworte: Almoraviden, Aoudaghost, Bilad al-Sudan, Kharidjismus, Malekismus

\begin{abstract}
The essay "On the Origins of Ideological Contradictions in the Sahel. The Almoravids in Western Bilad al-Sudan in the $11^{\text {th }}$ Century" determines the political and ideological reasons for the Almoravid expansion into the Bilad al-Sudan. The analysis of the Arab-Islamic narrative sources shows that the Almoravid advance into western Sudan was motivated, on the one hand, by the Almoravid emirs' intention to eliminate the Kharijite heresies and, on the other hand, by the goal of establishing a great Maliki empire in western Dar al-Islam. These ideological and political goals promoted the spread of an orthodox Islam. Until then, the Bilad al-Sudan was characterised by ethnic, social and religious diversity. Islam there was interspersed with heterodox tendencies: Shiism and especially Kharijism. The Almoravid movement accomplished this unification under the banner of triumphant Maliki islam.
\end{abstract}

Keywords: Almoravids, Aoudaghost, Bilad al-Sudan, Kharidjism, Malikism

\title{
Contact
}

contact de l'auteur/Autorkontakt/author contact: pare_mous@yahoo.fr contact de la revue/e-mail Zeitschrift/journal contact: redaktion.rcae@uni-bamberg.de journal homepage : https://ubp.uni-bamberg.de/rcae/index.php/rcae

Moussa PARÉ est maître de conférences en histoire médiévale et chef de département d'histoire à l'Université Félix Houphouët-Boigny (UFHB) à Abidjan (Côte d'Ivoire). Ses recherches portent sur la cour royale en occident chrétien et sur la ville et les espaces publics dans le Bilad al-Sudan.

Moussa PARÉ ist Hochschuldozent für mittelalterliche Geschichte und Leiter der Abteilung für Geschichte an der Université Félix Houphouët-Boigny (UFHB) in Abidjan (Côte d'Ivoire/Elfenbeinküste). Seine Forschungsschwerpunkte sind der Königshof im christlichen Westen des Mittelalters sowie die Stadt und die öffentlichen Räume im mittelalterlichen Bilad al-Sudan.

Moussa PARÉ is reader in medieval history and head of the history department at the Université Félix Houphouët-Boigny (UFHB) in Abidjan (Côte d'Ivoire/Ivory Coast). His research focuses on the royal court in the Christian West in the Middle Ages and on the city and public spaces in the medieval Bilad al-Sudan. 


\section{Introduction}

La première phase de l'islamisation s'est achevée au début du VIII ${ }^{\mathrm{e}}$ siècle en Afrique du Nord. Les populations Berbères longtemps réticents à l'égard de l'islam, finissent par adopter la nouvelle religion. Par l'intermédiaire des caravaniers nomades, cette religion s'est répandue peu à peu vers le sud. Mais, c'est à partir du XI $\mathrm{XI}^{\mathrm{e}}$ siècle que la seconde phase de l'islamisation se met en place avec le mouvement almoravide ${ }^{1}$ à la fois religieux et militaire. Les raisons de la poussée almoravide dans le Sudan occidental ont fait l'objet de spéculations. Deux thèses s'opposent :

La plus répandue est celle qui énonce que les Almoravides ont entrepris la conquête du Ghana dans le but d'imposer l'islam au peuple Soninké animiste. Cette thèse est défendue par Mahamadou Coulibaly qui, dans son article «L'attaque du Ghana XIe siècle», insiste sur les motifs religieux de l'attaque de Ghana par les troupes almoravides. Le territoire de Ghana a été le théâtre d'un véritable conflit confessionnel qui dura plusieurs années au $\mathrm{XI}^{\mathrm{e}}$ siècle, sous formes d'attaques et de raids menés par les Almoravides. Les prosélytes musulmans c'est-à-dire les Almoravides étant entrés en conflit avec une monarchie et un État dont le culte national était l' « animisme $»^{2}$.

La seconde thèse soutient que l'attaque du Ghana par les Almoravides participe de motifs économiques. Le contrôle du commerce de l'or est la raison fondamentale qui a poussé les Almoravides à conquérir le Ghana. Parmi les tenants de cette seconde thèse, on a Jean Boulègue qui, dans son article intitulé « Le mouvement almoravide (1048-1147) », indique que la première campagne c'est-à-dire, celle d'Aoudaghost menée par les Almoravides (1054-1055) aboutit à les rendre maitres des

\footnotetext{
${ }^{1}$ Les Almoravides sont une confédération de tribus berbères sanhadja (les Lemtuna, les Djodalla et les Massufa) originaires de l'Adrar. Ils nomadisaient entre l'actuel Sénégal et le sud du Maroc. Au XI ${ }^{\mathrm{e}}$ siècle, ils ont constitué un empire englobant l'ouest du Sahara, la partie occidentale du Maghreb et une partie de la péninsule Ibérique. Ils sont adeptes de l'idéologie malékite.

${ }^{2}$ Mahamadou Coulibaly : L'attaque de Ghana au XI ${ }^{\mathrm{e}}$ siècle, in : Afrika Zamani. Revue d'histoire africaine 2 (1974), p. 55-77, p. 61.
} 
voies commerciales et du commerce de l'or en provenance de la partie occidentale du Sudan au détriment du souverain soninké qui jusqu'à là en avait le monopole ${ }^{3}$. Jacques Maquet abonde dans le même sens. Il soutient que la production de l'or et d'autres marchandises précieuses sont à l'origine de grands courants de commerce contrôlés par le souverain de Ghana ${ }^{4}$. D'où la convoitise des lointains voisins du Maghreb et du Sahara occidental. Ainsi, les émirs almoravides prétextant répandre l'islam, entreprirent la conquête de la partie occidentale du Bilad alSudan pour tirer profit des richesses.

Au-delà de ces facteurs religieux et économique, nous pensons qu'il y a des fondements idéologiques et politiques à l'incursion almoravide dans cette zone qu'il convient d'analyser à la lumière des sources narratives arabo-musulmanes, notamment les chroniques historiques et les récits des géographes et voyageurs arabes ${ }^{5}$.

\section{Les Almoravides et l'expansion de l'idéologie malékite ${ }^{6}$}

À la fin du VIII ${ }^{\mathrm{e}}$ siècle, la ville de Ghana est un foyer de la propagation kharidjite. C'est à partir du VIII ${ }^{\mathrm{e}}$ siècle que les premiers missionnaires

\footnotetext{
${ }^{3}$ Jean BOULEGUE: Art. «Mouvement Almoravide», in : Encyclopadia Universalis [en ligne], consulté le 7 novembre 2021 :

https://www.universalis.fr/encyclopedie/mouvement-almoravide/ .

${ }^{4}$ Jean MAQUET : Les civilisations noires. Histoire-techniques-arts-sociétés, Paris 1962, p 220.

${ }^{5}$ Kitab al-Istibsar la chronique anonyme, Kitab al-Bayan d'Ibn Idhari al-Marrakushi, Routh el-Kartas d'Ibn Abi Zar, Kitab al-Ibar et Histoire des Berbères et des dynasties musulmanes de l'Afrique septentrionale d'Ibn Khaldun, le Kitab Surat al-ard d'Ibn Hawqal, le Kitab al-Masalik wa l-Mamalik d'al-Bakri.

${ }^{6}$ Le Malékisme est l'une des principales écoles (madhab-s) du droit musulman (fiqh) sunnite, se référant à l'œuvre du juriste médinois Malik ibn Anas (795). Elle est surtout implantée en Afrique occidentale, en Haute Egypte, au Soudan, en Arabie et concerne le quart des sunnites. Elle accorde une large place au Coran, à la Sunna, à la coutume, mais peu de place en revanche à la raison, ce qui conduit à une interprétation littérale des sources. Elle diffère essentiellement des trois autres écoles par les sources qu'elle utilise pour déterminer la jurisprudence. Si les quatre écoles utilisent le Coran, la sunna, ainsi que l'ijma (le consensus des experts) et les analogies (qiyas), le malékisme utilise également les pratiques des habitants de Médine (Amal ahl al-medina) comme sources de la jurisprudence (fiqh).
} 
kharidjites s'installent dans la ville de Ghana. Dans la seconde moitié du $\mathrm{XI}^{\mathrm{e}}$ siècle, la création du quartier musulman dans la ville est le résultat d'une présence ancienne. Selon al-Bakri :

Ghana se compose de deux villes situées dans une plaine. Celle qui est habitée par les musulmans est très grande et renferme douze mosquées, dans une desquelles on célèbre la prière le vendredi. Toutes ces mosquées ont leurs imams, leurs muezzins et des lecteurs salariés. La ville possède des jurisconsultes et des hommes remplis d'érudition?

Traqués par les Omeyyades qui ont fait du califat une monarchie temporelle, ces «protestants» doivent se réfugier dans la clandestinité ou s'enfuir $^{8}$. Vaincus dans la partie orientale de l'empire et harcelés en Arabie du Sud, les kharidjites ${ }^{9}$ se répandent au Maghreb et dans le Bilad alSudan, espaces bien plus favorables à leur prédication. Après le désastre de la bataille de Nahrawan (17 juillet 658), l'agitation kharidjite ne prend pour autant fin. Les révoltes qui éclatent à Basra et Kufa sont réprimées dans le sang. L'insurrection d'Abd Allahibn az-Zubayr, ayant pris le Hidjaz et La Mecque et obtenu des ralliements en Iraq, en Syrie et en Egypte est rendue moins effective par la présence d'éléments kharidjites dans certains des territoires que le petit-fils d'Abu Bakr cherche à soumettre. Cette présence kharidjite ne facilite pas la tâche des Omeyyades, qui sont

\footnotetext{
${ }^{7}$ AL-BAKRI : Livre des itinéraires et des royaumes, in : Recueil des sources arabes concernant l'Afrique Occidentale du VIII ${ }^{\mathrm{e}}-\mathrm{XVI}^{\mathrm{e}}$ siècle (Bilad al-Sudan), éd. Joseph Cuoq, Paris 1975, p 99.

${ }^{8}$ Eric Nogbou: Les relations politiques et religieuses entre le Maghreb al-Aqsa et l'Empire soninké du Ghana de l'avènement des Midrarites (772) au califat de Yaqub alMansour (1184-1199), Thèse de doctorat unique (soutenue le 08 mai 2010 sous la direction du Professeur Simon-Pierre Ekanza), Université de Cocody-Abidjan, p. 255.

${ }^{9}$ Le Kharidjisme est mouvement doctrinal et politique, né dans l'opposition à Ali. Il se caractérise par son rigorisme et son égalitarisme. Les kharidjites (en arabe khawāridj) sont les adeptes d'une secte musulmane qui remonte aux origines de l'islam et qui a joué un grand rôle d'opposition sous les califes omeyyades de Damas ; leur zèle s'est manifesté, sous des formes déjà moins violentes, pendant l'époque ' abbaside, surtout en Afrique du Nord. Ils furent progressivement réduits et maîtrisés, et ils ne forment plus aujourd'hui que des communautés disséminées au milieu des pays sunnites, mais jalouses de leur originalité. Néanmoins, tout au long de l'histoire du monde musulman, le kharidjisme n'a cessé de jouer un rôle : il a survécu, en dehors de son existence de secte, sous la forme d'une mentalité et d'un idéal. C'est souvent en se réclamant de lui que des populations se sont révoltées contre un pouvoir officiel qui les opprimait; ce fut le cas au Maghreb, où le nationalisme berbère trouva un appui dans le kharidjisme.
} 
obligés de réagir sur deux fronts. Le calife Marwan ibn al-Hakam (683685) réussit à conjurer le danger kharidjite en Syrie, puis en Egypte, où il nomme comme gouverneur son fils Abd al-Aziz. Son fils et successeur Abd al-Malik reprend Kufa en 694. Son général al-Hadjdjadj fait son entrée à La Mecque en 693, après un siège de six mois. Les kharidjites partent pour l'Iraq et le Far, où ils sèment la terreur, et il faut l'intervention d'al-Hadjdjadj, nommé gouverneur d'Iraq en 694, pour en venir à bout en 698-699.

Ainsi, après s'être solidement implantés au Maghreb à la chute de la dynastie omeyyade de Damas, les kharidjites migrent dans l'empire « animiste » de Ghana. Plus «démocratique» et «plus égalitaire», le kharidjisme apparaît, sous les traits d'un rigorisme moral, comme un ennemi des concessions et des compromissions inhérentes à l'exercice $\mathrm{du}$ pouvoir politique en Arabie. Ainsi, avant la grande vague sunnite du $\mathrm{XI}^{\mathrm{e}}$ siècle, menée par les Malékites almoravides, l'islam est fortement implanté dans la grande ville de Ghana.

Le Ghana devient ainsi un enjeu pour les États kharidjites de Tahert ${ }^{10}$ et de Sidjilmassa. Cette présence kharijite a un impact sur les populations soudanaises. Les premiers convertis sont évidemment leurs partenaires commerciaux. C'est donc aux kharijites que revient incontestablement le mérite d'être les pionniers de l'islamisation du Sudan.

Toutefois, dans la première moitié du $\mathrm{XI}^{\mathrm{e}}$ siècle, un mouvement réformateur orchestré par les tribus sanhadja du Sahara Occidental s'impose. Ce mouvement conduit par ibn Yasin mène une guerre sans merci contre les hérétiques berbères. La dimension idéologique devient donc un élément fondamental de la révolution almoravide :

Ce sont ces tribus qui après 440/1048 lancèrent l'appel pour la Vérité, la réparation des injustices et l'abolition des taxes injustes. Ce sont des sunnites, qui suivent l'école de

\footnotetext{
${ }^{10}$ Tahert est une principauté ibadite fondée par Ibn Rustem, ancien lieutenant au service des émirs Aghlabides de Kairouan. A partir, de 780, il prend son autonomie vis-à-vis des Aghlabides, alliés des Abbassides en Ifriqiya. Il fonde en 786 l'imamat de Tahert. La prise de Tahert par les Fatimides marque la fin de la dynastie rustémide.
} 
Malick b. Anas. Celui qui leur ouvrit la voie, les appela au ribat et à l'action pour la Vérité fut Abd Allah b. Yasin ${ }^{11}$.

La naissance du mouvement almoravide et l'instauration d'une politique de conquête entraînent des confrontations idéologiques avec les communautés kharidjites installées dans le Bilad al-Sudan. Les émirs almoravides, fervents adeptes de l'école malékite ${ }^{12}$, ne pouvaient tolérer plus longtemps l'influence grandissante de l'islam kharidjite dans le Sahara berbère et dans le Sudan. La première manifestation de cette confrontation idéologique est l'épineuse question d', sur la ville fondée au début du $\mathrm{X}^{\mathrm{e}}$ siècle par la grande famille des Sanhadja. Gouvernée par des chefs Lemtuna d'obédience sunnite dont l'autorité s'étendait sur des tribus du Sahara occidental et sur certains royaumes vassaux de Ghana ${ }^{13}$, le royaume d'Aoudaghost est conquis à la fin du $\mathrm{X}^{\mathrm{e}}$ siècle par les souverains animistes de Ghana avec le soutien des grands marchands kharidjites.

Pour les Berbères, l'idéologie kharidjite apparaît comme l'expression de leur opposition au sunnisme abbasside. A l'opposition politique contre la tutelle des califes d'Orient, se conjuguent donc des rivalités idéologiques. Les Berbères ont plus que besoin d'étendre l'influence kharidjite. Ceci leur permet de bouter hors du Maghreb l'idéologie sunnite, considérée à leurs yeux comme le symbole de l'oppression arabe.

C'est donc sur le terrain idéologique que se concentre un pan essentiel de l'expansion almoravide. Cette première manifestation de ces luttes entre les deux principales tendances de l'islam dans le Bilad al-Sudan est marquée par la reconquête d'Aoudaghost par les émirs almoravides :

An 446 (1054-1056 J-C), Ibn Yasin marcha sur Aoudaghast, pays florissant (...) c'était la résidence d'un roi nègre qui portait le titre de Ghana, avant que les Arabes

\footnotetext{
${ }^{11}$ AL-BAKRI : Livre des itinéraires et des royaumes, p. 86.

${ }^{12}$ La doctrine malikite (al-madhab al maliki) est fondée par Malik b. Anas (m. 796). Cette école juridique fait partie des quatre doctrines du monde musulman. Elle côtoie le hanafisme, le chafiisme et le hanbalisme.

${ }^{13}$ D'après AL-BAKRI : Livre des itinéraires et des royaumes, p. 311 : Telles sont les tribus qui, postérieurement à l'an 400 (1048-1049 de J.C.), entreprirent de maintenir la vérité, de réprimer l'injustice et d'abolir tous les impôts (qui n'étaient pas basés sur la loi). Elles professent la doctrine orthodoxe et suivent le rite institué par Malek Ibn Anas. Celui qui fraya cette voie et qui appela les peuples au Ribat et au maintien de la vérité se nommait Abd Allah Ibn Yasin.
} 
eussent pénétrés dans la ville de ce nom ... Les Almoravides emportèrent cette ville d'assaut, violèrent les femmes et s'emparèrent de tout ce qui s'y trouvaient en déclarant que s'était du butin légal (...) Les Almoravides traitèrent la population d'Aoudaghast avec cette rigueur extrême, parce qu'elle reconnaissait l'autorité du souverain de Aoudaghast ${ }^{14}$.

L'acharnement des émirs almoravides à vouloir reprendre Aoudaghost des mains des princes soninkés qui l'avaient annexée au $\mathrm{X}^{\mathrm{e}}$ siècle, découle également du fait que pour Ibn Yasin et ses troupes, il était inconcevable que leurs frères Sanhadja soient sous l'autorité d'un roi «animiste », allié des zenata kharidjites ${ }^{15}$. Pourtant, le kharidjisme au Ghana est un islam de commerçants dont les limites sont évidentes. Son champ de diffusion est très étroit et ne peut toucher les peuples profondément attachés à la religion traditionnelle et vers qui rien ne dénote une volonté particulière d'accepter le message de l'islam. Au contraire, on assiste à une persistance de la religion traditionnelle malgré plus de trois siècles de présence kharidjite.

Les marchands kharidjites ont, au départ, certainement privilégié leurs intérêts économiques au détriment des considérations religieuses. De plus, l'islam apparaît, pour les princes Soninkés, comme une religion qui risque de renverser l'ordre établi en mettant fin aux traditions ancestrales soninkés, fondement essentiel de l'existence même de la dynastie des Tounka. D'où le refus de cette religion par certains princes soninkés. En effet une mutation culturelle aussi profonde que l'islamisation, qui implique une certaine « répudiation des ancêtres, un renoncement à des coutumes et une coupure sociale suscite une certaine appréhension ${ }^{16}$.

Le $\mathrm{XI}^{\mathrm{e}}$ siècle marque un tournant dans les luttes idéologiques entre Kharidjites et Malékites dans le Bilad al-Sudan. La naissance du mouvement almoravide et l'instauration de sa politique du djihad le confrontent à ses voisins du Sud. Le mouvement almoravide est un mouvement de réforme islamique pour l'établissement de l'« islam originel ». C'est

\footnotetext{
${ }^{14}$ AL-BAKRI : Livre des itinéraires et des royaumes, p. 317.

${ }^{15}$ Vincent LAGARDÈRE : Les Almoravides, Paris 1998, p. 61.

${ }^{16}$ Eric NogBou/P.-C. N'CHO: Déclin religieux et déconstruction de la vie politique dans le Bilad al-Sudan. Une interprétation de la chute de l'empire soninké du Ghana (1077-1203), in : Revue ivoirienne d'histoire 34 (2019), p. 114-124.
} 
dans cette perspective que s'inscrivent les conquêtes almoravides. Selon Al-Zurhi, autrefois, les habitants étaient des infidèles, mais en l'an 469 (1076-1077), ils sont devenus de bons musulmans sous l'influence des Lamtouna ${ }^{17}$.

Cette nouvelle donne est marquée par une lutte pour l'éradication des hérésies dans la ville de Ghana. Les Berbères Sanhadja, fondateurs du mouvement almoravide, n'ont pas succombé aux sirènes du kharidjisme zenata. Le djihad almoravide paraît avoir, selon J. Cuoq, marqué profondément la mentalité religieuse et lui avoir insufflé ce rigorisme et cet attachement à la Loi islamique ${ }^{18}$. Ce djihad almoravide tient en partie à la situation géographique du pays dans le monde musulman. C'est l'islam tourné largement vers le monde soudanais animiste. Il est tout naturellement invité à l'expansion, à la conquête, au djihad ${ }^{19}$.

L'islamisation du Ghana est un facteur de déstabilisation du Soudan Occidental. Désormais, convertis à l'islam malékite au détriment des doctrines kharidjites, des princes soninkés cherchent maintenant à fonder un vaste Empire musulman à l'idéologie malékite dans le Soudan Occidental.

\section{L'établissement d'un État malékite dans le Sudan occidental}

La conversion à l'islam de certaines élites politiques à Ghana montre que ces princes ont trouvé dans l'islam la dimension religieuse qu'ils n'ont pas pu trouver dans les religions traditionnelles. De ce point de vue, la satisfaction des besoins religieux favorise la montée en puissance d'une élite à la recherche d'un renouveau politique où l'islam apparaît comme le fondement du nouvel État. Les engagements religieux prennent une direction nouvelle. Dès lors, le déclin de la religion traditionnelle menace les fondements de l'État soninké en produisant une alliance entre le politique et le religieux. Les mutations religieuses intensifient les passions politiques et favorisent une résurgence des idéologies

\footnotetext{
${ }^{17}$ Al-Zurhi, Djughrafiya, in: Recueil des sources arabes concernant l'Afrique Occidentale du VIII -XVI $^{\mathrm{e}}$ siècle (Bilad al-Sudan), éd. Joseph CuOQ, Paris 1975, p. 119.

${ }^{18}$ Joseph Cuoq : Histoire de l'islamisation de l'Afrique de l'Ouest, Paris 1984, p. 110.

${ }^{19}$ Joseph CuOQ : Histoire de l'islamisation de l'Afrique de l'Ouest, p. 110.
} 
révolutionnaires qui réclament l'exercice du pouvoir impérial. Les individus qui ont rompu avec la religion traditionnelle sont de potentielles recrues de l'idéologie malékite :

Les Mulaththamum [= les Almoravides] envahirent alors les Sudan, s'emparèrent de leurs défenses et de leur pays ... . Ils en amenèrent un grand nombre à embrasser l'islam comme religion ${ }^{20}$.

L'effondrement des structures religieuses traditionnelles, symbole du pouvoir soninké, entraîne une reconstruction politique du Soudan Occidental. Cette reconstruction favorise la naissance d'un État musulman avec la conversion à l'idéologie malékite d'une partie de l'élite politique soudanaise :

Ils devinrent musulmans au temps des Lamtuna et se distinguèrent dans leur islam. Ils sont aujourd'hui musulmans. Ils ont des ulama ${ }^{21}$, des fakih ${ }^{22}$, des lecteurs (de Coran). Ils excellent en tout cela. Quelques-uns d'entre eux sont venus en Andalousie, (c'étaient) des chefs de leurs notables. Ils ont fait le voyage de la Mekke, $y$ ont accompli le pèlerinage, ont fait la visite (au tombeau du prophète), sont revenus en leurs pays et ont dépensé beaucoup d'argent dans la guerre sainte ${ }^{23}$.

Fervents partisans de l'école d'al-Malik, les nouveaux souverains musulmans de Ghana poursuivent la politique de djihad prônée par les Malékites Almoravides. Convertie à l'idéologie almoravide, la dynastie des Touré hérite de la politique d'expansion initiée par les émirs almoravides. Le but de cette politique d'expansion agressive est avant tout de fonder un Empire malékite soudanais, consacrant ainsi la prééminence de l'Orthodoxie sunnite sur les « hérésies » chiites et kharidjites. Dès lors,

\footnotetext{
${ }^{20}$ IBn KHALDUN : Prolégomènes, in: Recueil des sources arabes concernant l'Afrique Occidentale du VIII ${ }^{\mathrm{e}}$-XVI ${ }^{\mathrm{e}}$ siècle (Bilad al-Sudan), éd. Joseph CuOQ, Paris 1975, p. 343.

${ }^{21}$ Les ulamas ou oulémas sont ceux qui possèdent le $̂$ lm, c'est-à-dire le savoir au sens le plus noble: Dieu et Sa loi. Ils jouent un grand rôle dans la cité. Ils exercent diverses fonctions (cadi, imam, prédicateur, docteur), auxquelles ils sont nommés par le prince ou dont ils héritent. Ils forment un groupe très institutionnalisé qui assure la transmission et la diffusion des sciences religieuses. Ils assurent aussi le prestige de la ville et son caractère islamique.

22 Jurisconsulte, savant en sciences religieuses, légiste. Un faqih est un expert en fiqh, ou, jurisprudence islamique. C'est donc un faqih est un expert en droit islamique, et, comme tel, le mot Faqih peut littéralement être traduit généralement par des juristes.

${ }^{23}$ Al-ZuHRI : Djughrafiya, p. 119.
} 
dans la première moitié $d u \mathrm{XII}^{\mathrm{e}}$ siècle, une véritable guerre sainte est lancée contre les États animistes du Soudan Occidental d'une part, et contre les États musulmans non orthodoxes d'autre part :

Il $y$ a dans leur territoire, une tribu qui habite le bord de la grande Mer, à l'Ouest, ceux-ci sont des infidèles qui suivent la religion des Madjous ${ }^{24}$. Personne n'entre dans leur pays et aucune marchandise n'y est importée. Les gens de Ghana envahissent chaque année leur territoire. Ces gens ne connaissent pas le fer et combattent avec des barres en bois d'ébène; les gens de Ghana peuvent les vaincre parce qu'ils combattent avec des épées et des lances ${ }^{25}$.

Les justifications du djihad sont à dominante défensive: il s'agit de protéger les musulmans et les dhimmis, de préserver l'intégrité territoriale du Dar al-islam, de développer la coopération dans tous les États de l'islam pour étendre davantage le message prophétique, d'établir et de renforcer la loi islamique, d'aider les Musulmans sous domination des non-musulmans et d'assurer le respect des traités et l'allégeance (aman) des non-musulmans ${ }^{26}$. Les souverains du Ghana s'affichent comme un rempart contre les agressions du Dar al-Harb, au-delà des régions sous leur administration directe. Il semble que la doctrine du djihad défensif ait été adoptée par les souverains du Ghana, ce qui n'empêche pas pour autant les razzias.

\section{Conclusion}

Au-delà du souci d'étendre l'islam et du désir de contrôler le commerce de l'or, l'expansion almoravide dans le Bilad al-Sudan occidental participe de motivations idéologiques et politiques marquées par une politique d'éradication des hérésies kharidjites et la formation d'un vaste empire d'obédience malékite. La construction d'un Empire musulman par les Almoravides dont les frontières couvrent les deux rives du Sahara est source de graves tensions qui aboutissent, à la fin du XII ${ }^{\mathrm{e}}$ siècle, à la disparition de l'Empire des Cissé Tounkara où se sont installés très tôt les

\footnotetext{
${ }^{24}$ Le culte traditionnel.

${ }^{25}$ Al-Zurhi : Djughrafiya, p 120.

${ }^{26}$ Rémi DeWIÈrE : Un Etat islamique en paroles, in : Du lac Tchad à la Mecque : le sultanat de Borno et son monde (XVI ${ }^{\mathrm{e}}$-XVII ${ }^{\mathrm{e}}$ siècle), Paris 2017, p. 297-325.
} 
kharidjites. La révolution incarnée par les Almoravides qui prétend restaurer la foi authentique des premiers temps de l'islam, a contribué ainsi à en construire un modèle, qui n'a jamais cessé d'évoluer. 


\section{Références bibliographiques}

\section{Sources}

AL-BAKRI : Livre des itinéraires et des royaumes, in : Recueil des sources arabes concernant l'Afrique Occidentale du VIII ${ }^{\mathrm{e}}$-XVI ${ }^{\mathrm{e}}$ siècle (Bilad al-Sudan), éd. Joseph CuOQ, Paris : CNRS 1975, pp. 82-123.

AL-ZURHI : Djughrafiya, in : Recueil des sources arabes concernant l'Afrique Occidentale du VIII ${ }^{\mathrm{e}}$-XVI ${ }^{\mathrm{e}}$ siècle (Bilad al-Sudan), , éd. Joseph CuOQ, Paris : CNRS 1975, p. 115-123.

IBN KHALDUN : Prolégomènes, in : Recueil des sources arabes concernant l'Afrique Occidentale du VIII ${ }^{\mathrm{e}}$ XVI $^{\mathrm{e}}$ siècle (Bilad al-Sudan), , éd. Joseph CuOQ, Paris : CNRS 1975, p. 328-363.

\section{Études}

BOUlEGUE Jean : Art. « Mouvement Almoravide », in : Encyclopædia Universalis [en ligne], consulté le 7 novembre 2021.

URL : https://www.universalis.fr/encyclopedie/mouvement-almoravide/.

Coulibaly, Mahamadou: L'attaque de Ghana au XIe siècle, in : Afrika Zamani. Revue d'histoire africaine 2 (1974), p. 55-77.

Cuoq, Joseph : Histoire de l'islamisation de l'Afrique de l'Ouest, Paris : Librairie Orientaliste Paul Geuthner 1984.

Dewiere, Rémi : Un État Islamique En Paroles, In : Du Lac Tchad À La Mecque : Le Sulta-

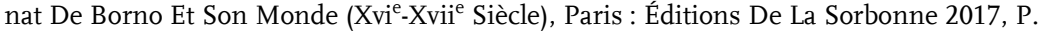
297-325.

LAGARDERE, Vincent : Les Almoravides, Paris : L’Harmattan 1998.

MAQUET, Jean : Les civilisations noires. Histoire - techniques - arts - sociétés, Paris : Horizon de France 1962.

NoGbou, Eric : Les relations politiques et religieuses entre le Maghreb al-Aqsa et l'Empire soninké du Ghana : de l'avènement des Midrarites (772) au califat de Yaqub al-Mansour (1184-1199), Thèse de doctorat unique (soutenue le 08 mai 2010 sous la direction du Professeur Simon-Pierre Ekanza), Université de Cocody (Abidjan/Côte d'Ivoire) 2010.

Nogbou, M. Eric/N'CHO P.-C. : Déclin religieux et déconstruction de la vie politique dans le Bilad al-Sudan. Une interprétation de la chute de l'empire soninké du Ghana (1077-1203), in : Revue ivoirienne d'histoire 34 (2019), p. 114-124. 